OUTP-03-08P

gr-qc/0303082

\title{
Surface terms and the Gauss-Bonnet Hamiltonian
}

\author{
Antonio Padilla* \\ Theoretical Physics, Department of Physics, \\ University of Oxford, 1 Keble Road, Oxford OX1 3NP, U.K.
}

\begin{abstract}
We derive the gravitational Hamiltonian starting from the Gauss-Bonnet action, keeping track of all surface terms. This is done using the language of orthonormal frames and forms to keep things as tidy as possible. The surface terms in the Hamiltonian give a remarkably simple expression for the total energy of a spacetime. This expression is consistent with energy expressions found in hep-th/0212292. However, we can apply our results whatever the choice of background and whatever the symmetries of the spacetime.
\end{abstract}

*a.padilla1@physics.ox.ac.uk 


\section{Introduction}

The energy of gravitational systems has attracted much interest down the years (see, for example $[1,2,3,4,5,6,7,8,9,10,11,12,13,14,15]$ ). In particular, for Einstein gravity

$$
S=\kappa \int R-2 \Lambda
$$

expressions were found for the energy of asymptotically flat [1] and asymptotically AdS [2] spacetimes. Some time later, Hawking and Horowitz [5] gave a general derivation of the gravitational Hamiltonian, keeping careful track of surface terms. When evaluated on a solution, this Hamiltonian gave an expression for the total energy. This agreed with the earlier expressions found in 1,22. However, this new expression could be applied regardless of the asymptotic behaviour of the solution.

Recently, Deser and Tekin have found energy expressions for higher derivative gravities [14,15]. This inital work has focused on test spacetimes that are asymptotically maximally symmetric, with background spacetimes (vacua), defined to be maximally symmetric everywhere. In this paper, we will perform the analogue of Hawking and Horowitz's calculation by deriving the Hamiltonian for Gauss-Bonnet (GB) gravity: a special, but important class of higher derivative gravities. We will obtain an expression for the energy by once again evaluating this Hamiltonian on a solution. As with [5], our expression will be consistent with earlier results [14, 15], but can be easily applied even if the background is not maximally symmetric. This greater flexibility allows for a more natural choice of vacua in some cases, as we will illustrate with an example.

Before going any further, let us briefly mention what GB gravity is, and why it is important. GB gravity is given by the addition of the Gauss-Bonnet invariant to the Einstein-Hilbert action (11),

$$
S=\kappa \int R-2 \Lambda+\alpha\left(R^{2}-4 R_{A B} R^{A B}+R_{A B C D} R^{A B C D}\right) .
$$

In $D=4$ dimensions, the GB term is a topological invariant and does not enter the dynamics. This ceases to be the case in $D>4$ dimensions. Furthermore, in Einstein gravity, the vacuum field equation are given by a linear combination of the Einstein tensor and the metric. In four dimensions, this is the most general combination of tensors that satisfies the following conditions [16]:

- it is symmetric.

- it depends only on the metric and its first two derivatives.

- it has vanishing divergence.

- it is linear in the second derivatives of the metric ${ }^{1}$.

\footnotetext{
${ }^{1}$ In $D=4$, this condition is actually implied by the other three.
} 
However, it has become unfashionable to restrict ourselves to just four dimensions. If we go to $D=5$ or 6 , it turns out that these conditions are satisfied by a linear combination of the metric, the Einstein tensor, and the Lovelock tensor [16,17]. The Lovelock tensor arises from the variation of the Gauss-Bonnet term in the above action (2). As $D$ increases, we can use the same arguments to introduce higher order invariants (the Euler characters) [18. In this sense, GB gravity is the natural generalisation of Einstein gravity to 5 or 6 dimensions, although other higher derivative gravity theories have also been studied (see, for example [19, 20, 21, 22, 23]).

It is interesting to note that the GB action (2) is renormalisable [24], but perhaps the most compelling reason to study GB gravity is its appearance in String theory. Consider the slope (ie. $\alpha^{\prime}$ ) expansion for the heterotic string. At lowest order, it is given by the Einstein-Hilbert action (11). The first stringy corrections give rise to quadratic curvature terms [25, 26]. For this effective theory to be ghost-free, the quadratic curvatures must be in the combination given in the GB action (2) [27. 28. This link to String theory has generated a lot of research into higher derivative gravity and cosmology [29, 30, 31, 32, 33, 34, 35, 36, 37, 38, 39, 40, 41, 42, 43, 44, 45, 46, 47, 48, 49, 50]. Although the early work [29, 30, 31, 32, 33] achieved reduction to four dimensions via Kaluza-Klein compactifications, more recent studies [34, 35, 36, 37, 38, 39, 40, 41,42, 43, 44, 45, 46, 47, 48,49,50] have focussed on the braneworld scenario [51,52]. Braneworld models are themselves inspired by String theory [53] so it is natural to ask what effect any stringy corrections might have on their cosmology. From a holographic point of view, we might expect such higher curvature terms in the bulk to correspond to next to leading order corrections in the $1 / N$ expansion of the CFT on the boundary/brane 43. Calculating the GB Hamiltonian will allow us to investigate the GB version of "exact" braneworld holography [54,55]. This will be discussed in a future article [56].

The rest of this paper is organised as follows: in section 2 we will give a derivation of the GB Hamiltonian, with extra details given in the appendix. We will use orthonormal frames and differential forms to keep things as tidy as possible. Some readers may wish to ignore the details of this derivation and go directly to the energy expression at the end of the section. In section 3 we will show that this expression is consistent with existing literature [57,14,15]. We will illustrate the flexibility of our approach with a special example in section 3.2. Section 4 contains some concluding remarks.

\section{Derivation of the Gauss-Bonnet Hamiltonian}

\subsection{The action}

The Gauss-Bonnet action (2) is most elegantly written in terms of differential forms. Suppose our $D$-dimensional spacetime, $\mathcal{M}$, has metric,

$$
g=\eta_{A B} E^{A} \otimes E^{B}
$$

where $\left\{E^{A}\right\}$ is an orthonormal basis of 1-forms, and indices are raised/lowered using $\eta_{A B}=\operatorname{diag}(-1,+1, \ldots,+1)$. We write $\left\{X_{A}\right\}$ for the dual basis of vectors. 
We will find it useful to define the following forms,

$$
e_{A_{1} \ldots A_{m}}=\frac{1}{(D-m) !} \epsilon_{A_{1} \ldots A_{m} A_{m+1} \ldots A_{D}} E^{A_{m+1}} \wedge \ldots \wedge E^{A_{D}}
$$

where $\epsilon_{A_{1} \ldots A_{D}}$ is the totally antisymmetric tensor with $\epsilon_{0 \ldots(D-1)}=1$. Notice that the scalar $D$-form, $e$, is the volume measure on $\mathcal{M}$.

Now suppose we have vanishing torsion, and that $\omega^{A}{ }_{B}$ is the connection 1-form compatible with the metric $g$. The curvature 2 -form is given by

$$
\Omega_{B}^{A}=d \omega_{B}^{A}+\omega_{C}^{A} \wedge \omega_{B}^{C}=\frac{1}{2} R_{B C D}^{A} E^{C} \wedge E^{D} .
$$

The right hand equation above gives the Riemann tensor, $R_{B C D}^{A}$. The Ricci tensor is then defined by $R_{B D}=R_{B C D}^{C}$ and the Ricci scalar by $R=\eta^{A B} R_{A B}$. The GB action (2) can now be written

$$
S=\kappa \int_{\mathcal{M}}-2 \Lambda e+\Omega^{A B} \wedge e_{A B}+\alpha \Omega^{A B} \wedge \Omega^{C D} \wedge e_{A B C D}
$$

where we have made use of the following identity [18, 22,

$$
E^{B} \wedge e_{A_{1} \ldots A_{m}}=\delta_{A_{m}}^{B} e_{A_{1} \ldots A_{m-1}}-\delta_{A_{m-1}}^{B} e_{A_{1} \ldots A_{m-2} A_{m}}+\ldots+(-1)^{m-1} \delta_{A_{1}}^{B} e_{A_{2} \ldots A_{m}},
$$

If $\mathcal{M}$ has a boundary, $\partial \mathcal{M}$, we need to define boundary conditions on $\partial \mathcal{M}$. We usually demand that the geometry of the boundary is fixed. However, as it stands, the action (6) is inconsistent with these boundary conditions. This is because its variation with respect to the metric does not vanish on shell.

To cure this, we need to add a boundary integral. In Einstein gravity, this is the well known Gibbons-Hawking term [58]. The generalisation to higher derivative gravities was worked out by Myers [18. For GB gravity it is given by

$$
S_{\text {boundary }}=-\kappa \int_{\partial \mathcal{M}} \theta^{A B} \wedge e_{A B}+2 \alpha \theta^{A B} \wedge\left(\Omega^{C D}-\frac{2}{3} \theta_{E}^{C} \wedge \theta^{E D}\right) \wedge e_{A B C D},
$$

where $\theta^{A B}$ is the second fundamental form [59,60]. It is defined as

$$
\theta^{A B}=\omega^{A B}-\omega_{0}^{A B} .
$$

where $\omega_{0}^{A B}$ is the connection for the product metric, $g_{0}$, that agrees with $g$ on the boundary. The second fundamental form is closely related to the extrinsic curvature of $\partial \mathcal{M}$ in $\mathcal{M}[18$.

The fully consistent action for Gauss-Bonnet gravity is therefore given by,

$$
S=S_{\text {bulk }}+S_{\text {boundary }}
$$

where $S_{\text {bulk }}$ and $S_{\text {boundary }}$ are given by equations (6) and (8) respectively. It is interesting to note that the boundary integrand contains a linear combination of the first 
two Chern-Simons forms. This is related to the fact that the bulk integrand contains the same linear combination of the first two Euler characters [18.

Finally, we note that although the action (10) is well defined for spatially compact manifolds, it is divergent when $\mathcal{M}$ is spatially noncompact [5]. To get round this we need to choose a reference background, $\overline{\mathcal{M}}$ with metric $\bar{g}$. This background should be a static solution to the field equations [5], but does not have to be maximally symmetric. The boundary conditions are unchanged which means that $\partial \overline{\mathcal{M}}$ should have the same geometry as $\partial \mathcal{M}$. We can then define the background action, $\bar{S}$, in the same way as before. The physical action is the difference,

$$
I=\Delta S=S-\bar{S}
$$

In the Hamiltonian picture, this background can be thought of as defining a background energy or zero energy solution. For example, for an asymptotically AdS spacetime, we would probably choose the background to be pure AdS, but we do not have to. Any asymptotically AdS black hole spacetime would be equally valid.

\subsection{Splitting space and time}

Although the physical Hamiltonian will be constructed from the action (11), it is clear that it will just be the difference of the Hamiltonian constructed from $S$ and that constructed from $\bar{S}[\underline{5}$. For the time being we will concentrate on the former.

\subsubsection{Foliations of $\mathcal{M}$}

To proceed, we need to deconstruct the spacetime $\mathcal{M}$ by separating space from time in the following way. First, we choose a timelike vector field, $\partial / \partial t$. Now introduce a family of spacelike hypersurfaces $\left\{\Sigma_{t}\right\}$ labelled by the parameter $t$. This family is a foliation of the full spacetime. We assume that the hypersurfaces have no inner boundaries and do not intersect each other. They meet the timelike part of the boundary (call this $B$ ) orthogonally, and in the far past/future, they coincide with the spacelike part of the boundary (call this $\Sigma_{\infty}$ ). Therefore the total boundary, $\partial \mathcal{M}=B \cup \Sigma_{\infty}$.

We can write the metric for $\mathcal{M}$ in $\mathrm{ADM}$ form [1],

$$
g=-N^{2} d t^{2}+\gamma_{a b}\left(t, x^{a}\right)\left(d x^{a}+N^{a} d t\right)\left(d x^{b}+N^{b} d t\right)
$$

where $N$ is the lapse function, $N^{a}$ the shift vector, and $\gamma_{a b}\left(t, x^{a}\right)$ the induced metric on $\Sigma_{t}$. It is natural to choose the following orthonormal basis of 1 -forms,

$$
E^{\perp}=N d t, \quad E^{a}=E^{a}{ }_{b}\left(d x^{b}+N^{b} d t\right)
$$

where $\delta_{a b} E^{a}{ }_{c} E^{b}{ }_{d}=\gamma_{c d}$. We would like to emphasize some notation at this point. Lowercase latin indices label components in $\Sigma_{t}$, whereas uppercase latin indices label components in $\mathcal{M}$. For example, $\left\{E^{a}\right\}$ is an orthonormal basis for $\Sigma_{t}$ whereas $\left\{E^{A}\right\}=$ $E^{\perp} \cup\left\{E^{a}\right\}$ is an orthonormal basis for $\mathcal{M}$. 
The dual basis of vectors is given by 61

$$
X_{\perp}=\frac{1}{N}\left(\frac{\partial}{\partial t}-N^{a} \frac{\partial}{\partial x^{a}}\right), \quad X_{a}=E_{a}{ }^{b} \frac{\partial}{\partial x^{a}} .
$$

$X_{\perp}$ is the vector normal to $\Sigma_{t}$, and is not necessarily equal, or even parallel to $\partial / \partial t$. We should also note that $X_{\perp}$ is tangent to $B$, as $\Sigma_{t}$ and $B$ are orthogonal.

Normally, the next step is to use the Gauss-Codazzi equations 62 to rewrite the bulk part of the action (see for example [5]). This has infact been done for GB gravity [63] although the contribution from surface terms was ignored. In this paper we are using the language of orthonormal frames and differential forms. We therefore need to know how to translate the Gauss-Codazzi equations into this language. This is explained in 64, so we will merely state their results.

The Gauss-Codazzi equations describe the decomposition of the bulk Riemann tensor into spatial tensors defined on $\Sigma_{t}$. In the language of forms, it is the curvature 2 -form that we wish to decompose. We start by decomposing the connection, $\omega^{A B}$.

$$
\begin{aligned}
\omega^{\perp a} & =-H^{a}+a^{a} E^{\perp}, \\
\omega^{a b} & =\tilde{\omega}^{a b}+l^{a b} E^{\perp} .
\end{aligned}
$$

Here, we have two 0-forms: a vector, $a^{a}$, and an antisymmetric tensor, $l^{a b}$. The 1form $H^{a}=H_{b}^{a} E^{b}$, where $H_{b}^{a}$ is the extrinsic curvature of $\Sigma_{t}$ in $\mathcal{M}$. From now on, anything labelled with a tilde is intrinsic to $\Sigma_{t}$, as opposed to $\mathcal{M}$. Therefore, $\tilde{\omega}^{a b}$ is the connection for the induced metric $\gamma=\delta_{a b} E^{a} \otimes E^{b}$.

We now write the Gauss-Codazzi equations in the following way

$$
\begin{aligned}
\Omega^{\perp a} & =-\tilde{\nabla} H^{a}+E^{\perp} \wedge\left[-\$_{\perp} H^{a}-\frac{1}{N} \tilde{\nabla}\left(N a^{a}\right)-l^{a b} H_{b}\right] \\
\Omega^{a b} & =\tilde{\Omega}^{a b}+H^{a} \wedge H^{b}+E^{\perp} \wedge\left[\$_{\perp} \tilde{\omega}^{a b}-\frac{1}{N} \tilde{\nabla}\left(N l^{a b}\right)+H^{a} a^{b}-H^{b} a^{a}\right]
\end{aligned}
$$

where $\tilde{\Omega}^{a b}=\tilde{d} \tilde{\omega}^{a b}+\tilde{\omega}^{a}{ }_{c} \wedge \tilde{\omega}^{c b}$ is the curvature 2-form for $\Sigma_{t}$, and the operator $\tilde{\nabla}$ is the covariant exterior derivative [59,60] (on $\Sigma_{t}$ ). Note that $\tilde{\nabla}$ reduces to the exterior derivative, $\tilde{d}$, when acting on scalars. The definition of the operator $\$_{\perp}$ is given in 64 . It is closely related to the Lie derivative with respect to the vector $X_{\perp}$, although it lives entirely on $\Sigma_{t}$ and acts on tensor components as if they were scalars. In many ways it behaves like a partial derivative ${ }^{2}$.

In principle we could also decompose the torsion 2-form (see 64]). However, we have set torsion to zero, which means that every component of the torsion decomposition must vanish. This gives the following conditions:

$$
\begin{aligned}
H^{a b} & =H^{b a}, \\
a_{a} E^{a} & =\frac{\tilde{d} N}{N}, \\
\tilde{\nabla} E^{a} & =0 \\
\$_{\perp} E^{a} & =-H^{a}-l_{b}^{a} E^{b} .
\end{aligned}
$$

\footnotetext{
${ }^{2}$ It is often useful to think of $\$_{\frac{\partial}{\partial t}}$ as the frame-form version of $\frac{\partial}{\partial t}$.
} 


\subsubsection{Foliations of $B$}

Since we intend to keep careful track of surface terms, we will need a foliation of $B$, as well as $\mathcal{M}$. On $B$, the foliation is given by the family of surfaces $\left\{S_{t}\right\}$. For a given value of $t, S_{t}$ is the intersection of $B$ and $\Sigma_{t}$.

We need to understand how bulk quantities project on to $S_{t}$. Near $S_{t}$, the metric can be written in Gaussian normal coordinates

$$
g=d z^{2}-E^{\perp} \otimes E^{\perp}+\delta_{i j} E^{i} \otimes E^{j}
$$

From now on we will write $E^{z}$ for the extension of $d z$ into the bulk, and $X_{z}$ for the extension of the inward pointing normal $\partial / \partial z$. Notice that we are using indices $i, j$ etc to label components in $S_{t}$.

We can use the techniques developed in 64 to project bulk quantities on to $B$ and then on to $S_{t}$. We find that the decomposition of the connection is given by

$$
\begin{aligned}
\omega^{\perp z} & =b_{i} E^{i}+c E^{\perp} \\
\omega^{\perp i} & =-\widehat{H}^{i}+a^{i} E^{\perp}+b^{i} E^{z} \\
\omega^{z i} & =\widehat{K}^{i}+b^{i} E^{\perp} \\
\omega^{i j} & =\widehat{\omega}^{i j}+l^{i j} E^{\perp}+\chi^{i j} E^{z}
\end{aligned}
$$

where $\chi^{i j}$ is some antisymmetric tensor, and

$$
\begin{aligned}
c & =a^{z} \\
b^{i} & =l^{z i}=-H^{z i}
\end{aligned}
$$

Note that anything wearing a hat is intrinsic to $S_{t}$. Therefore, $\widehat{\omega}^{i j}$ is the connection for the induced metric (on $S_{t}$ ), $\lambda=\delta_{i j} E^{i} \otimes E^{j}$. We have also defined,

$$
\begin{aligned}
\widehat{H}^{i} & =H^{i}{ }_{j} E^{j}, \\
\widehat{K}^{i} & =K^{i}{ }_{j} E^{j} .
\end{aligned}
$$

Here, we should interpret $H^{i}{ }_{j}$ and $K^{i}{ }_{j}$ as the extrinsic curvatures of $S_{t}$ in $B$ and $\Sigma_{t}$ respectively. 
The curvature decomposition is given by

$$
\begin{aligned}
& \Omega^{\perp z}=\widehat{d}\left(b_{i} E^{i}\right)+\widehat{H}^{i} \wedge \widehat{K}_{i}+E^{z} \wedge\{\cdots\} \\
& +E^{\perp} \wedge\left[\widehat{\Phi}_{\perp}\left(b_{i} E^{i}\right)-\frac{1}{N} \widehat{d}(N c)-a^{i} \widehat{K}_{i}-b^{i} \widehat{H}_{i}\right] \\
& \Omega^{\perp i}=-\widehat{\nabla} \widehat{H}^{i}+b_{j} E^{j} \wedge \widehat{K}^{i}+E^{z} \wedge\{\cdots\} \\
& +E^{\perp} \wedge\left[-\widehat{\$}_{\perp} \widehat{H}^{i}-\frac{1}{N} \widehat{\nabla}\left(N a^{i}\right)-l^{i j} \widehat{H}_{j}+c \widehat{K}^{i}-b^{i} b_{j} E^{j}\right] \\
& \Omega^{z i}=\widehat{\nabla} \widehat{K}^{i}-b_{j} E^{j} \wedge \widehat{H}^{i}+E^{z} \wedge\{\cdots\} \\
& +E^{\perp} \wedge\left[\widehat{\$}_{\perp} \widehat{K}^{i}-\frac{1}{N} \widehat{\nabla}\left(N b^{i}\right)+\widehat{K}_{j} l^{i j}-b_{j} E^{j} a^{i}-c \widehat{H}^{i}\right] \\
& \Omega^{i j}=\widehat{\Omega}^{i j}+\widehat{H}^{i} \wedge \widehat{H}^{j}-\widehat{K}^{i} \wedge \widehat{K}^{j}+E^{z} \wedge\{\cdots\} \\
& +E^{\perp} \wedge\left[\widehat{\$}_{\perp} \widehat{\omega}^{i j}-\frac{1}{N} \widehat{\nabla}\left(N l^{i j}\right)+2 \widehat{H}^{[i} a^{j]}+2 \widehat{K}^{[i} b^{j]}\right]
\end{aligned}
$$

where we will not care what is contained in $\{\cdots\}$ as the integration of $E^{z}$ over $B$ is zero. In analogy with the previous section, $\widehat{\Omega}^{i j}$ and $\widehat{\nabla}$ are the curvature form and covariant exterior derivative on $S_{t}$, respectively. Again, $\widehat{\nabla}$ reduces to the exterior derivative, $\widehat{d}$, when acting on scalars. The operator $\widehat{\Phi}_{\perp}$ is the analogue of $\$_{\perp}$ on $B^{3}$.

As long as we are near $B$, equations (32) to (35) are the frame-form version of the Gauss-Codazzi equations for a hypersurface of codimension two [65].

\subsection{The Hamiltonian}

We are now ready to start calculating the Hamiltonian. However, we will find it convenient to continue working with the action until virtually the bitter end. When our action finally has the desired form we will switch to the Hamiltonian picture, and give an expression for the gravitational energy of a solution.

We will start with the bulk part of the action (6). Our aim is to write it so that it contains no derivatives of the lapse function or the shift vector. This is because these are ignorable coordinates, and should behave like Lagrange multipliers. They will be paired with the Hamiltonian and momentum constraints respectively, as is the case in Einstein gravity [5]. We also want to eliminate terms like $\$_{\perp} H^{a}$, which contain second time derivatives of the canonical variable $E^{a}$. We will need to use integration by parts to achieve these aims. This means that the bulk action (6) will contribute surface terms. In summary, we expect to write (6) as

$$
S_{\mathrm{bulk}}=S_{\mathrm{bulk}}^{*}+S_{\text {leftover }}
$$

where $S_{\text {leftover }}$ are the leftover surface terms, and

$$
S_{\text {bulk }}^{*}=\kappa \int d t \int_{\Sigma_{t}} \pi_{a} \wedge \dot{E}^{a}-N \mathcal{H}-N^{a} \mathcal{H}_{a}
$$

${ }^{3}$ If $\tilde{A}$ is an arbitrary $p$-form in $\Sigma_{t}$, then near $S_{t}$, we can write $\tilde{A}=\widehat{A}+A_{z} \wedge E^{z}$, where $\widehat{A}$ and $A_{z}$ are $p$ and $(p-1)$-forms respectively, living on $S_{t}$. It can then be shown that $\Phi_{\perp} \tilde{A}=\widehat{\Phi}_{\perp} \widehat{A}+E^{z} \wedge\{\cdots\}$. 
Here $\pi_{a}$ is the momentum conjugate to $E^{a}$, and $\mathcal{H}$ and $\mathcal{H}_{a}$ are the Hamiltonian and momentum constraints respectively. We have also introduced the intuitive notation $\dot{E}^{a}=\$_{\frac{\partial}{\partial t}} E^{a}$.

At this stage, the surface part of the action is given by equation (8). We can split this into two parts,

$$
S_{\text {boundary }}=S_{B}+S_{\infty}
$$

where $S_{B}$ contains the integration over $B$ and $S_{\infty}$ the integration over $\Sigma_{\infty}$. In section 2.5. we will group these terms with $S_{\text {leftover }}$. This will give us a modified boundary term

$$
S_{\text {boundary }}^{*}=S_{\text {boundary }}+S_{\text {leftover }} \text {. }
$$

which will be closely related to the gravitational energy of a solution.

\subsection{The bulk}

As promised, we start with the bulk part of the action (6), in the hope of deriving

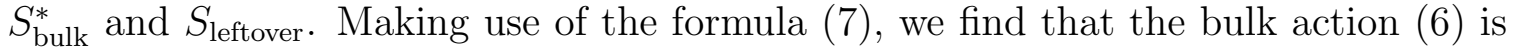
given by

$$
S_{\text {bulk }}=S_{\text {kinetic }}+S_{1}+S_{2}+S_{3}
$$

where

$$
\begin{aligned}
S_{\text {kinetic }}=\kappa \int_{\mathcal{M}} E^{\perp} \wedge\left\{-2 \$_{\perp} H^{b} \wedge \zeta_{b}\right. & \left.-4 \alpha\left[\tilde{\nabla} H^{b} \wedge \$_{\perp} \tilde{\omega}^{c d}+\$_{\perp} H^{b} \wedge F^{c d}\right] \wedge \zeta_{b c d}\right\} \\
S_{1}= & \kappa \int_{\mathcal{M}} E^{\perp} \wedge\left\{-2 \Lambda \zeta+F^{a b} \wedge \zeta_{a b}+\alpha F^{a b} \wedge F^{c d} \wedge \zeta_{a b c d}\right\} \\
S_{2}= & \kappa \int_{\mathcal{M}} E^{\perp} \wedge\left\{-\frac{2}{N} \tilde{\nabla}\left(N a^{b}\right) \wedge \zeta_{b}\right. \\
S_{3}= & \kappa \int_{\mathcal{M}} E^{\perp} \wedge\left\{4 \alpha\left[\tilde{\nabla} H^{b} \wedge \frac{1}{N} \tilde{\nabla}\left(N \tilde{l}^{c d}\right)-l^{b a} H_{a} \wedge F^{c d}\right] \wedge \zeta_{b c d}\right\} .
\end{aligned}
$$

with

$$
F^{a b}=\tilde{\Omega}^{a b}+H^{a} \wedge H^{b}
$$

Note that we have introduced the analogue of $e_{A_{1} \ldots A_{m}}$ on $\Sigma_{t}$,

$$
\zeta_{a_{1} \ldots a_{m}}=e_{\perp a_{1} \ldots a_{m}}=\frac{1}{(D-1-m) !} \epsilon_{a_{1} \ldots a_{m} a_{m+1} \ldots a_{D-1}} E^{a_{1}} \wedge \ldots \wedge E^{a_{D-1}} .
$$


We will now rewrite expressions (41) to (44), bearing in mind the goals we mentioned at the beginning of this section. The kinetic term is given by

$$
\begin{aligned}
S_{\text {kinetic }}= & \kappa \int d t \int_{\Sigma_{t}} \pi_{a} \wedge \dot{E}^{a}-N^{a} \mathcal{H}_{a} \\
& +\kappa \int d t \int_{S_{t}} 4 \alpha N H^{b} \wedge \$_{\perp} \tilde{\omega}^{c d} \wedge \zeta_{b c d}+(-1)^{D} N^{a} \pi_{b} E^{b}{ }_{a}-S_{\infty} .
\end{aligned}
$$

where

$$
\begin{aligned}
\pi_{a} & =-2 \zeta_{a b} \wedge H^{b}-4 \alpha \zeta_{a b c d} \wedge H^{b} \wedge \Lambda^{c d} \\
\Lambda^{c d} & =\tilde{\Omega}^{c d}+\frac{1}{3} H^{c} \wedge H^{d}
\end{aligned}
$$

and the Hamiltonian constraint,

$$
\mathcal{H}_{a}=(-1)^{(D-1)} E^{b}{ }_{a} \tilde{\nabla} \pi_{b} .
$$

In section 2.6, we will show that $\pi_{a}$ is indeed the momentum conjugate to $E^{a}$. The derivation of equation (47) is given in the appendix.

The remaining terms in the bulk action can be written as follows:

$$
\begin{aligned}
S_{1} & =-\kappa \int d t \int_{\Sigma_{t}} N \mathcal{H} \\
S_{2} & =\kappa \int d t \int_{S_{t}} N\left[2 a^{b} \zeta_{b}+4 \alpha a^{b} F^{c d} \wedge \zeta_{b c d}\right] \\
S_{3} & =-\kappa \int d t \int_{S_{t}} 4 \alpha N \tilde{\nabla} H^{b} l^{c d} \wedge \zeta_{b c d}
\end{aligned}
$$

where the Hamiltonian constraint, $\mathcal{H}$, is given by

$$
\mathcal{H}=2 \Lambda \zeta-F^{a b} \wedge \zeta_{a b}-\alpha F^{a b} \wedge F^{c d} \wedge \zeta_{a b c d}
$$

Note that in deriving the expression (53) for $S_{3}$, we made use of the following:

$$
\begin{gathered}
\tilde{\nabla}^{2} H^{b}=\tilde{\Omega}_{c}^{b} \wedge H^{c} . \\
H_{a} \wedge\left[\tilde{l}^{b a} F^{c d}+l^{c d} \tilde{\Omega}^{b a}\right] \wedge \zeta_{b c d}=0 .
\end{gathered}
$$

Collecting together equations (47), (51), (52), and (53), we see that $S_{\text {bulk }}$ takes the desired form

$$
S_{\mathrm{bulk}}=S_{\mathrm{bulk}}^{*}+S_{\text {leftover }}
$$

with $S_{\text {bulk }}^{*}$ given by equation (37) and $S_{\text {leftover given by }}$

$$
\begin{aligned}
S_{\text {leftover }}=-S_{\infty}+ & \kappa \int d t \int_{S_{t}}(-1)^{D} N^{a} \pi_{b} E^{b}{ }_{a} \\
& +N\left\{2 a^{b} \zeta_{b}+4 \alpha\left[H^{b} \wedge \$_{\perp} \tilde{\omega}^{c d}+a^{b} F^{c d}-\tilde{\nabla} H^{b} l^{c d}\right] \wedge \zeta_{b c d}\right\} .
\end{aligned}
$$




\subsection{The boundary}

As expected, rewriting the bulk part of the action in the desired form (37) has altered the boundary part of the action. In particular, we have a leftover surface integral (58) that must be added to the original boundary part of the action (38). This gives the modified boundary action,

$$
S_{\text {boundary }}^{*}=S_{\text {leftover }}+S_{\infty}+S_{B}
$$

In order to combine each term in the above equation, we need to write them in a common form. This will involve integrations over $S_{t}$, of well defined quantities on $S_{t}$.

Let us begin with $S_{\text {leftover }}+S_{\infty}$. From (23) we know that near $B, E^{z}{ }_{a}=\delta^{z}{ }_{a}$ and $N^{z}=0$. This means that the momentum term in (158) gives

$$
\kappa \int d t \int_{S_{t}}(-1)^{D} N^{a} \pi_{b} E^{b}{ }_{a}=\kappa \int d t \int_{S_{t}}(-1)^{D} N^{i} \pi_{j} E^{j}{ }_{i} .
$$

We now use the fact that

$$
\$_{\perp} \tilde{\omega}^{z i}=\widehat{\$}_{\perp} \widehat{K}^{i}+E^{z}\{\cdots\}
$$

along with (28) and (29) to rewrite the remaining terms. We find that

$$
\begin{aligned}
& S_{\text {leftover }}+S_{\infty}=\kappa \int d t \int_{S_{t}}(-1)^{D} N^{i} \pi_{j} E^{j}{ }_{i}+2 N c \phi \\
& +4 \alpha N\left[-b_{k} E^{k} \wedge \widehat{\$}_{\perp} \widehat{\omega}^{i j}+c F^{i j}+F^{z} l^{i j}-2 \widehat{H}^{i} \wedge \widehat{\$}_{\perp} \widehat{K}^{j}-2 a^{i} F^{z j}-2 F^{i} b^{j}\right] \wedge \phi_{i j}
\end{aligned}
$$

where $F^{b}=-\tilde{\nabla} H^{b}$. We have also introduced the $S_{t}$ analogue of $\zeta_{a_{1} \cdots a_{n}}$ and $e_{A_{1} \cdots A_{n}}$ :

$$
\phi_{i_{1} \cdots i_{n}}=\zeta_{z i_{1} \cdots i_{n}}=e_{\perp z i_{1} \cdots i_{n}} .
$$

Meanwhile, terms like $F^{z}$ and $F^{i j}$ can be deduced by comparing equations (32) to (35) with (17) and (18).

$$
\begin{aligned}
F^{z} & =\widehat{d}\left(b_{k} E^{k}\right)+\widehat{H}^{k} \wedge \widehat{K}_{k} \\
F^{i} & =-\widehat{\nabla} \widehat{H}^{i}+b_{k} E^{k} \wedge \widehat{K}^{i} \\
F^{z j} & =\widehat{\nabla} \widehat{K}^{j}-b_{k} E^{k} \wedge \widehat{H}^{j} \\
F^{i j} & =\widehat{\Omega}^{i j}+\widehat{H}^{i} \wedge \widehat{H}^{j}-\widehat{K}^{i} \wedge \widehat{K}^{j}
\end{aligned}
$$

Note that we have dropped all terms like $E^{z}\{\cdots\}$ as we are now integrating over $B$.

Now consider $S_{B}$. The only non-zero components of $\theta^{A B}$ on $B$ are:

$$
\theta^{\perp z}=\omega^{\perp z}, \quad \theta^{z i}=\omega^{z i}
$$


where $\omega^{\perp z}$ and $\omega^{z i}$ are given by equations (24) and (26) respectively. We use this fact, along with equations (33) and (35), to write $S_{B}$ in the following way:

$$
\begin{aligned}
& S_{B}= \kappa \int d t \int_{S_{t}} 2 N \widehat{K}^{i} \wedge \phi_{i}-2 N c \phi+4 \alpha N \widehat{K}^{i} \wedge\left[F^{j k}+\frac{2}{3} \widehat{K}^{j} \wedge \widehat{K}^{k}\right] \wedge \phi_{i j k} \\
&+ 4 \alpha N\left\{-2 b^{i}\left[F^{j}-\frac{2}{3} b_{k} E^{k} \wedge \widehat{K}^{j}\right]+2 \widehat{K}^{i} \wedge\left[G^{j}+\frac{2}{3}\left(-c \widehat{K}^{j}+b^{j} b_{k} E^{k}\right)\right]\right. \\
&\left.-c\left[F^{i j}+\frac{2}{3} \widehat{K}^{i} \wedge \widehat{K}^{j}\right]+b_{k} E^{k} \wedge\left[G^{i j}+\frac{4}{3} b^{[i} \widehat{K}^{j]}\right]\right\} \wedge \phi_{i j}
\end{aligned}
$$

where

$$
\begin{aligned}
G^{j} & =-\widehat{\$}_{\perp} \widehat{H}^{j}-\frac{1}{N} \widehat{\nabla}\left(N a^{j}\right)-l^{j k} \widehat{H}_{k}+c \widehat{K}^{j}-b^{j} b_{k} E^{k} \\
G^{i j} & =\widehat{\$}_{\perp} \widehat{\omega}^{i j}-\frac{1}{N} \widehat{\nabla}\left(N l^{i j}\right)+2 \widehat{H}^{[i} a^{j]}+2 \widehat{K}^{[i} b^{j]}
\end{aligned}
$$

The expressions (62) and (69) now have a common form, so we can combine them to get

$$
S_{\text {boundary }}^{*}=S_{\text {boundary }, 1}+S_{\text {boundary }, 2}+S_{\text {boundary }, 3}
$$

where

$$
\begin{gathered}
S_{\text {boundary }, 1=}=\kappa \int d t \int_{S_{t}}(-1)^{D} N^{i} \pi_{j} E^{j}{ }_{i}+N\left\{2 \widehat{K}^{i} \wedge \phi_{i}\right. \\
\left.+4 \alpha \widehat{K}^{i} \wedge\left[\widehat{\Omega}^{j k}+\widehat{H}^{j} \wedge \widehat{H}^{k}-\frac{1}{3} \widehat{K}^{j} \wedge \widehat{K}^{k}\right] \wedge \phi_{i j k}\right\} \\
S_{\text {boundary }, 2=}=\kappa \int d t \int_{S_{t}} 4 \alpha N\left\{\widehat{d}\left(b_{k} E^{k}\right) l^{i j}-\frac{1}{N} b_{k} E^{k} \wedge \widehat{\nabla}\left(N l^{i j}\right)\right. \\
\left.-2 a^{i} \widehat{\nabla} \widehat{K}^{j}-\frac{2}{N} \widehat{K}^{i} \wedge \widehat{\nabla}\left(N a^{j}\right)\right\} \wedge \phi_{i j} \\
S_{\text {boundary }, 3=}=\kappa \int d t \int_{S_{t}} 4 \alpha N\left\{-2 \widehat{H}^{i} \wedge \widehat{\Phi}_{\perp} \widehat{K}^{j}-2 \widehat{K}^{i} \wedge \widehat{\Phi}_{\perp} \widehat{H}^{j}\right. \\
\left.+\widehat{H}^{k} \wedge \widehat{K}_{k} l^{i j}-2 \widehat{K}^{i} \wedge l^{j k} \widehat{H}_{k}\right\} \wedge \phi_{i j}
\end{gathered}
$$

Since we have zero torsion, we use the condition

$$
\widehat{\nabla} \phi_{i_{1} \cdots i_{n}} \equiv 0
$$

to show that the integrand in $S_{\text {boundary,2 }}$ is a total derivative. Since $S_{t}$ is a boundary of $\Sigma_{t}$, it has no boundary of its own. This means that,

$$
S_{\text {boundary }, 2}=0
$$

By integrating by parts on the time derivatives in (75), we can also show that

$$
S_{\text {boundary }, 3}=\kappa \int d t \int_{S_{t}} 4 \alpha N\left\{-2 \widehat{K}^{i} \wedge \widehat{H}^{j} \wedge \widehat{H}^{k} \wedge \phi_{i j k}\right\} .
$$


Here we have used the zero torsion result

$$
\widehat{\$}_{\perp} E^{i}=-\widehat{H}^{i}-l_{j}^{i} E^{j} .
$$

and the identity

$$
\begin{aligned}
\widehat{H}^{k} \wedge \widehat{K}_{k} l^{i j} \wedge \phi_{i j}-2 \widehat{K}^{i} \wedge \widehat{H}_{k} l^{j k} \wedge \phi_{i j}-2 \widehat{K}^{i} \wedge \widehat{H}^{j} l^{k}{ }_{l} \wedge E^{l} \wedge \phi_{i j k} \\
=\widehat{K}^{i} \wedge E_{i} \wedge \widehat{H}^{j} l^{k l} \wedge \phi_{j k l}=0 .
\end{aligned}
$$

The last equality in (80) follows from the symmetry of $\widehat{K}^{i j}$.

We now collect together equations (73), (77) and (178) to deduce that,

$$
\begin{aligned}
S_{\text {boundary }}^{*}=\kappa \int d t \int_{S_{t}}(-1)^{D} N^{i} \pi_{j} E^{j}{ }_{i} & +N\left\{2 \widehat{K}^{i} \wedge \phi_{i}\right. \\
+4 \alpha \widehat{K}^{i} \wedge & {\left.\left[\widehat{\Omega}^{j k}-\widehat{H}^{j} \wedge \widehat{H}^{k}-\frac{1}{3} \widehat{K}^{j} \wedge \widehat{K}^{k}\right] \wedge \phi_{i j k}\right\} }
\end{aligned}
$$

\subsection{The conjugate momentum}

Now that we have the action in its correct form, it remains to calculate the momentum conjugate to $E^{a}$. This is given by,

$$
p_{a}=\frac{\partial \mathcal{L}_{\mathrm{bulk}}}{\partial \dot{E}^{a}}
$$

where $\mathcal{L}_{\text {bulk }}$ is the bulk integrand. We could take $\mathcal{L}_{\text {bulk }}$ from $S_{\text {bulk }}^{*}$. However, it is convenient to temporarily undo the integration by parts that gives equation (148) (see appendix). In other words, we leave the derivatives of $N^{a}$ in the bulk action. This is perfectly OK, because it does not affect the bulk dynamics, and therefore the value of the conjugate momentum. The bulk integrand is temporarily given by,

$$
\mathcal{L}_{\text {bulk }}=\pi_{a} \wedge N \$_{\perp} E^{a}-N \mathcal{H}
$$

where $\pi_{a}$ and $\mathcal{H}$ are given by equations (48) and (54) respectively. Using the zero torsion decomposition (22), we can say

$$
\mathcal{L}_{\text {bulk }}=-N \pi_{a} \wedge\left(H^{a}+l^{a}{ }_{b} E^{b}\right)-N \mathcal{H}=-N \pi_{a} \wedge H^{a}-N \mathcal{H}
$$

where the right hand equation follows from (149), and the antisymmetry of $l^{a b}$.

Referring to equations (22) and (146), we use the chain rule to show that,

$$
p_{a}=-\frac{1}{N} \frac{\partial \mathcal{L}_{\mathrm{bulk}}}{\partial \widehat{H}^{a}}=\pi_{a}
$$

This non-trivial result is due to the following cancelation,

$$
\frac{\partial \pi_{b}}{\partial \widehat{H}^{a}} \wedge \widehat{H}^{b}+\frac{\partial \mathcal{H}}{\partial \widehat{H}^{a}}=0 .
$$


We conclude that $\pi_{a}$ is indeed the conjugate momentum. It should be thought of as a function of $\dot{E}^{a}$. To derive the Hamiltonian, we should invert this function. However, $\pi_{a}$ is cubic in $\dot{E}^{a}$, so the inverse is multivalued. This is a well known property of higher derivative gravities. In the Hamiltonian picture, this could mean that we could jump from one solution to another. These "zigzagging" histories still provide an extremum of the action. In this paper, we will assume that at any given time, we have a unique solution. This is just the same as saying that we are not in the process of jumping from one solution to another. For more discussion on multivalued Hamiltonians in this context, refer to [63, 66].

\subsection{The physical Hamiltonian}

We have shown that we can write our action as,

$$
S=S_{\text {bulk }}^{*}+S_{\text {boundary }}^{*}=\kappa \int d t\left[\int_{\Sigma_{t}} \mathcal{L}_{\text {bulk }}^{*}+\int_{S_{t}} \mathcal{L}_{\text {boundary }}^{*}\right]
$$

where

$$
\begin{aligned}
\mathcal{L}_{\text {bulk }}^{*}= & \pi_{a} \wedge \dot{E}^{a}-N \mathcal{H}-N^{a} \mathcal{H}_{a} \\
\mathcal{L}_{\text {boundary }}^{*}=N\{ & \left.2 \widehat{K}^{i} \wedge \phi_{i}+4 \alpha \widehat{K}^{i} \wedge\left[\widehat{\Omega}^{j k}-\widehat{H}^{j} \wedge \widehat{H}^{k}-\frac{1}{3} \widehat{K}^{j} \wedge \widehat{K}^{k}\right] \wedge \phi_{i j k}\right\} \\
& +(-1)^{D} N^{i} \pi_{j} E^{j}{ }_{i} .
\end{aligned}
$$

The corresponding Hamiltonian is defined as,

$$
\begin{aligned}
H & =\kappa \int_{\Sigma_{t}} \pi_{a} \wedge \dot{E}^{a}-\mathcal{L}_{\text {bulk }}^{*}-\kappa \int_{S_{t}} \mathcal{L}_{\text {boundary }}^{*} \\
& =\kappa \int_{\Sigma_{t}} N \mathcal{H}+N^{a} \mathcal{H}_{a}-\kappa \int_{S_{t}} \mathcal{L}_{\text {boundary }}^{*}
\end{aligned}
$$

To arrive at the physical Hamiltonian, we need to subtract off the background Hamiltonian,

$$
\bar{H}=-\kappa \int_{S_{t}} \overline{\mathcal{L}}_{\text {boundary }}^{*}
$$

Here we have used the fact that the background is a stationary solution to the field equations [5],

$$
\overline{\mathcal{H}}=\overline{\mathcal{H}}_{a}=\bar{\pi}_{a}=0 .
$$

The physical Hamiltonian is therefore given by,

$$
H_{\text {phys }}=\kappa \int_{\Sigma_{t}} N \mathcal{H}+N^{a} \mathcal{H}_{a}-\kappa \int_{S_{t}} \Delta \mathcal{L}_{\text {boundary }}^{*}
$$

where

$$
\begin{aligned}
\Delta \mathcal{L}_{\text {boundary }}^{*}= & (-1)^{D} N^{i} \pi_{j} E^{j}{ }_{i}+N\left\{2 \Delta \widehat{K}^{i} \wedge \phi_{i}\right. \\
& \left.+4 \alpha\left[\Delta \widehat{K}^{i} \wedge\left(\widehat{\Omega}^{j k}-\widehat{H}^{j} \wedge \widehat{H}^{k}\right)-\frac{1}{3} \Delta\left(\widehat{K}^{i} \wedge \widehat{K}^{j} \wedge \widehat{K}^{k}\right)\right] \wedge \phi_{i j k}\right\}
\end{aligned}
$$


For any quantity $Q$ in the test spacetime with corresponding quantity $\bar{Q}$ in the background, $\Delta Q=Q-\bar{Q}$. Notice that we have $\Delta \widehat{\Omega}^{j k}=\Delta \widehat{H}^{j}=0$. This is because the geometry of the boundary is the same in the test spacetime, as in the background.

If our test spacetime is a solution to the field equations, it satisfies the constraints

$$
\mathcal{H}=\mathcal{H}_{a}=0
$$

Its energy is then given by the value of the physical Hamiltonian,

$$
\begin{aligned}
E=-\kappa \int_{S_{t}}( & -1)^{D} N^{i} \pi_{j} E^{j}{ }_{i}+N\left\{2 \Delta \widehat{K}^{i} \wedge \phi_{i}\right. \\
& \left.+4 \alpha\left[\Delta \widehat{K}^{i} \wedge\left(\widehat{\Omega}^{j k}-\widehat{H}^{j} \wedge \widehat{H}^{k}\right)-\frac{1}{3} \Delta\left(\widehat{K}^{i} \wedge \widehat{K}^{j} \wedge \widehat{K}^{k}\right)\right] \wedge \phi_{i j k}\right\}
\end{aligned}
$$

Given the technical complexity of Gauss-Bonnet gravity, we believe that this expression is remarkably simple. Note that for $\alpha=0$, we recover the correct result for Einstein gravity, as of course we should.

\subsection{Using a coordinate basis}

Although the final result (96) is neat and tidy, we might prefer to work in a coordinate basis, and express the Hamiltonian in terms of the familiar tensors of General Relativity. In this case, our canonical variable is the induced metric $\gamma_{a b}$. The conjugate momentum, $\pi^{a b}$, is given by [64],

$$
\pi^{a b} d^{D-1} x=\frac{1}{2} \pi^{a} \wedge E^{b} .
$$

With this in mind, we can verify that the Hamiltonian and momentum constraints given by equations (54) and (50) respectively, agree with the corresponding expressions in [63]. If $z^{A}$ is the normal to the timelike boundary, $B$, and $n^{A}$ the normal to $\Sigma_{t}$, the Hamiltonian (93) can be written

$$
H_{\text {phys }}=\kappa \int_{\Sigma_{t}} d^{D-1} x\left[N \mathcal{H}+N^{a} \mathcal{H}_{a}\right]-\kappa \int_{S_{t}} d^{D-2} x \Delta \mathcal{L}_{\text {boundary }}^{*}
$$

where now

$$
\begin{aligned}
\Delta \mathcal{L}_{\text {boundary }}^{*}= & 2 N^{i} \pi_{i j} z^{j}+N \sqrt{\lambda}\{2 \Delta K \\
& \left.+2 \alpha(3 !) \delta_{i}^{[l} \delta_{j}^{m} \delta_{k}^{n]}\left[\Delta K_{l}^{i}\left(\widehat{R}^{j k}{ }_{m n}-2 H_{m}^{j} H_{n}^{k}\right)-\frac{2}{3} \Delta\left(K_{l}^{i} K_{m}^{j} K_{n}^{k}\right)\right]\right\}
\end{aligned}
$$

Here, $\widehat{R}_{m n}^{j k}$ is the Riemann tensor on $S_{t}$, constructed out of the induced metric $\lambda_{i j}$. $K_{j}^{i}$ and $H_{j}^{i}$ are the extrinsic curvatures of $S_{t}$ in $\Sigma_{t}$ and $B$ respectively, and $K$ is the trace of $K_{j}^{i}$. More precisely,

$$
K_{i j}=-\lambda_{(i}^{l} \lambda_{j)}^{m} \nabla_{l} z_{m}, \quad H_{i j}=-\lambda_{(i}^{l} \lambda_{j)}^{m} \nabla_{l} n_{m}, \quad K=\lambda_{i}^{j} K_{j}^{i} .
$$




\section{Comparison with previous energy expressions}

\subsection{Deser and Tekin}

Now that we have derived an expression for the energy in GB gravity, we should compare it with previous results in the literature. In particular, Deser and Tekin [14, 15 used a "conserved charge" technique to derive the energy of asymptotically maximally symmetric spacetimes above backgrounds of constant curvature. This method can be applied to generic higher derivative gravities, but we can check it is consistent with our result in the Gauss-Bonnet case.

Suppose our test spacetime $\mathcal{M}$, is asymptotically maximally symmetric. We choose our background, $\overline{\mathcal{M}}$ to be the maximally symmetric solution with curvature form,

$$
\bar{\Omega}^{A B}=\frac{2 \Lambda_{\mathrm{eff}}}{(D-1)(D-2)} E^{A} \wedge E^{B}
$$

The effective cosmological constant is given by,

$$
\Lambda_{\mathrm{eff}}=-\frac{1}{4 \alpha^{*}}\left[1 \pm \sqrt{1+8 \Lambda \alpha^{*}}\right], \quad \alpha^{*}=\alpha \frac{(D-3)(D-4)}{(D-1)(D-2)} .
$$

which is real and multivalued for $1+8 \Lambda \alpha^{*}>0$.

Our aim is to calculate the energy associated with the timelike Killing vector $\partial / \partial t$. We can choose our foliation $\left\{\Sigma_{t}\right\}$ so that the shift vector vanishes. Since $\partial / \partial t$ is Killing, it is clear that $\widehat{H}^{i}=0$. The expression for the energy reduces to,

$$
E=-\kappa \int_{S_{t}} N\left\{2 \Delta \widehat{K}^{i} \wedge \phi_{i}+4 \alpha\left[\Delta \widehat{K}^{i} \wedge \widehat{\Omega}^{j k}-\frac{1}{3} \Delta\left(\widehat{K}^{i} \wedge \widehat{K}^{j} \wedge \widehat{K}^{k}\right)\right] \wedge \phi_{i j k}\right\}
$$

Since $S_{t}$ lies in the asymptotic region, we can say,

$$
\Delta\left(\widehat{K}^{i} \wedge \widehat{K}^{j} \wedge \widehat{K}^{k}\right) \wedge \phi_{i j k} \approx 3 \Delta \widehat{K}^{i} \wedge\left(\bar{K}^{j} \wedge \bar{K}^{k}\right) \wedge \phi_{i j k}
$$

where $\bar{K}^{j}$ is $\widehat{K}^{j}$ evaluated on the background. The energy is now given by,

$$
E=-\kappa \int_{S_{t}} N\left\{2 \Delta \widehat{K}^{i} \wedge \phi_{i}+4 \alpha \Delta \widehat{K}^{i} \wedge\left(\widehat{\Omega}^{j k}-\bar{K}^{j} \wedge \bar{K}^{k}\right) \wedge \phi_{i j k}\right\}
$$

Using the fact that $\bar{H}^{j}=0$, it is clear from equations (35) and (101) that,

$$
\widehat{\Omega}^{j k}-\bar{K}^{j} \wedge \bar{K}^{k}=\frac{2 \Lambda_{\mathrm{eff}}}{(D-1)(D-2)} E^{j} \wedge E^{k} .
$$

This implies that the energy,

$$
E=-\kappa\left(1+4 \alpha^{*} \Lambda_{\mathrm{eff}}\right) \int_{S_{t}} 2 N \Delta \widehat{K}^{i} \wedge \phi_{i}= \pm \kappa \sqrt{1+8 \Lambda \alpha^{*}} \int_{S_{t}} 2 N \Delta \widehat{K}^{i} \wedge \phi_{i}
$$

where we have used the $S_{t}$ analogue of the useful formula (7), and the cosmological constant relation (102). 
In order to make contact with [14,15, we switch to a coordinate basis,

$$
E= \pm \kappa \sqrt{1+8 \Lambda \alpha^{*}} \int_{S_{t}} d^{D-2} x \sqrt{\lambda} 2 N \Delta K
$$

We now follow the procedure described in [5] for Einstein gravity. Let us start with the test spacetime. Near $S_{t}$, we can express the metric on $\Sigma_{t}$ in Gaussian normal coordinates,

$$
d s_{\Sigma_{t}}^{2}=\gamma_{a b} d x^{a} d x^{b}=d z^{2}+q_{i j}\left(z, x^{k}\right) d x^{i} d x^{j}
$$

where $q_{i j}\left(0, x^{k}\right)=\lambda_{i j}$ is the metric on $S_{t}$. Similarly, for the background, we can write the metric on $\bar{\Sigma}_{t}\left(\right.$ near $\left.S_{t}\right)$, as,

$$
d s_{\bar{\Sigma}_{t}}^{2}=\bar{\gamma}_{a b} d \bar{x}^{a} d \bar{x}^{b}=d \bar{z}^{2}+\bar{q}_{i j}\left(\bar{z}, \bar{x}^{k}\right) d \bar{x}^{i} d \bar{x}^{j} .
$$

To ensure that the normals to $S_{t}$ agree on the test spacetime and the background, we choose the diffeomorphism $z=\bar{z}$ and $x^{i}=\bar{x}^{i}$. In these coordinates,

$$
K=-\frac{1}{2} q^{i j} q_{i j, z}, \quad \bar{K}=-\frac{1}{2} \bar{q}^{i j} \bar{q}_{i j, z}
$$

Since both metrics agree on the boundary, we note that $\Delta q_{i j}=0$ there. Therefore on $S_{t}$,

$$
\Delta K=-\frac{1}{2} \lambda^{i j}\left(\Delta q_{i j}\right)_{, z}=-\frac{1}{2}(\Delta q)_{, z}
$$

where $\Delta q=\lambda^{i j} \Delta q_{i j}$. This gives a final energy expression,

$$
E=\mp \kappa \sqrt{1+8 \Lambda \alpha^{*}} \int_{S_{t}} d^{D-2} x \sqrt{\lambda} N(\Delta q)_{, z} .
$$

We now use Deser and Tekin's method [14,15] to calculate ${ }^{4}$ the energy associated with the timelike Killing vector, $t^{A}$.

$$
\begin{array}{r}
E_{\mathrm{DT}}=\mp \kappa \sqrt{1+}+8 \Lambda \alpha^{*} \int_{S_{t}} d S_{A} n_{B}\left\{t_{C} \bar{\nabla}^{B} h^{A C}-t_{C} \bar{\nabla}^{A} h^{B C}+t^{B} \bar{\nabla}^{A} h-t^{A} \bar{\nabla}^{B} h\right. \\
\left.+h^{B C} \bar{\nabla}^{A} t_{C}-h^{A C} \bar{\nabla}^{B} t_{C}+t^{A} \bar{\nabla}_{C} h^{B C}-t^{B} \bar{\nabla}_{C} h^{A C}+h \bar{\nabla}^{B} t^{A}\right\}
\end{array}
$$

where $h^{A B}=g^{A B}-\bar{g}^{A B}$ and $h=\bar{g}_{A B} h^{A B}$. Here $g_{A B}$ and $\bar{g}_{A B}$ are the metrics on $\mathcal{M}$ and $\overline{\mathcal{M}}$ respectively. We will choose to work in a synchronous gauge for which $n^{A} h_{A B}=0$ [5]. As the metrics agree on the boundary we can also set $h^{A B}=0$ on $S_{t}$. If $S_{t}$ has inward pointing normal $\partial / \partial z$, the measure is given by $d S_{A}=-d^{D-2} x \sqrt{\lambda} \delta_{A}^{z}$. For vanishing shift vector, we have $t^{A}=N n^{A}$, and the energy (114) simplifies to,

$$
E_{\mathrm{DT}}= \pm \kappa \sqrt{1+8 \Lambda \alpha^{*}} \int_{S_{t}} d^{D-2} x \sqrt{\lambda} N\left(\partial_{b} h^{z b}-\partial^{z} h\right)
$$

\footnotetext{
${ }^{4}$ In [14 15, the authors do not explicitly write down an energy expression for the GB action with a bare cosmological constant $\Lambda$. However, they give enough information to easily derive equation (114).
} 
In the Gaussian Normal coordinates we have recently described, $h^{z b}=0$ and $h=\Delta q$. Deser and Tekin's energy now reads,

$$
E_{\mathrm{DT}}=\mp \kappa \sqrt{1+8 \Lambda \alpha^{*}} \int_{S_{t}} d^{D-2} x \sqrt{\lambda} N \partial^{z}(\Delta q) .
$$

This expression clearly agrees with the equation (113). We can conclude that although our derivation was very different to that in [14,15], our results are consistent.

\subsubsection{Application to GB black holes}

One of the nice features of GB gravity (2) is that it contains static, spherically symmetric solutions [67, 57, 68, 69, 70, 71, 49, 66] of the form,

$$
d s^{2}=-V(r) d t^{2}+\frac{d r^{2}}{V(r)}+r^{2} d \Omega_{D-2}^{2}
$$

where $d \Omega_{D-2}^{2}$ is the metric on a unit $(D-2)$-sphere. We will assume that $1+8 \Lambda \alpha^{*}>0$, so that there are two possible branches for the potential,

$$
V(r)=1+\frac{r^{2}}{2(D-1)(D-2) \alpha^{*}}\left(1 \pm \sqrt{1+8 \Lambda \alpha^{*}+\frac{4(D-1)(D-2) \alpha^{*} \mu}{r^{D-1}}}\right)
$$

Here $\mu \geq 0$ is a constant of integration that gives mass to the spacetime. The upper branch has a naked singularity at $r=0$, whereas the lower branch is a real black hole with a unique event horizon surrounding the singularity [57]. We wish to calculate the mass, $M$, of these spacetimes above the appropriate maximally symmetric background,

$$
d s^{2}=-\bar{V}(r) d t^{2}+\frac{d r^{2}}{\bar{V}(r)}+r^{2} d \Omega_{D-2}^{2}
$$

where

$$
\bar{V}(r)=1+\frac{r^{2}}{2(D-1)(D-2) \alpha^{*}}\left(1 \pm \sqrt{1+8 \Lambda \alpha^{*}}\right) .
$$

The foliation in each case is given by surfaces of constant $t$, so we soon see that,

$$
q_{i j}=r^{2}(z) \chi_{i j}, \quad \bar{q}_{i j}=\bar{r}^{2}(z) \chi_{i j}
$$

where $\chi_{i j}$ is the metric on the unit $(D-2)$-sphere, and

$$
\frac{d r}{d z}=-\sqrt{V}, \quad \frac{d \bar{r}}{d z}=-\sqrt{\bar{V}}
$$

If $r(0)=\bar{r}(0)=R$, on $S_{t}$, it follows that,

$$
(\Delta q)_{, z}=-\frac{2(D-2)}{R} \Delta \sqrt{V(R)} \approx-\frac{(D-2)}{R \sqrt{\bar{V}}} \Delta V(R) \quad \text { on } S_{t} .
$$


For large $R$,

$$
\Delta V(R) \approx \pm \frac{1}{\sqrt{1+8 \Lambda \alpha^{*}}} \frac{\mu}{R^{D-3}}
$$

Finally, we note that $N=\sqrt{V}$ and apply equation (113) to give,

$$
M=\kappa \Omega_{D-2}(D-2) \mu
$$

where $\Omega_{D-2}$ is the volume of the unit $(D-2)$-sphere. This is the standard result. It is always valid for $\Lambda_{\text {eff }} \leq 0$. For $\Lambda_{\text {eff }}>0$ our analysis is valid only if the de Sitter horizon is much larger than the black hole horizon [15].

\section{$3.2 \quad$ A special case}

In the last section, we assumed that $1+8 \Lambda \alpha^{*}>0$. Now consider what happens when $1+8 \Lambda \alpha^{*}=0$. We cannot make use of the expression (113) because it involves multiplying an infinite integral, by zero! We will not worry about how one would modify the approach of [14,15] to accomodate this. Instead, we will sell the approach developed in this paper. Let us focus on the 5-dimensional black hole with,

$$
V(r)=1+\frac{r^{2}}{4 \alpha}-\sqrt{\frac{\mu}{2 \alpha}} .
$$

To calculate the mass, we need to go back to equation (96). We also need to choose a background. In this example, the maximally symmetric solution with $\mu=0$ is not necessarily the most natural choice. We might prefer $\mu$ to be chosen so that the horizon has zero area [57]. Whatever our choice, we illustrate the flexibility of this work by allowing for non-maximally symmetric backgrounds. We will keeps things general and say that the background potential is given by

$$
\bar{V}(r)=1+\frac{r^{2}}{4 \alpha}-\sqrt{\frac{\bar{\mu}}{2 \alpha}} .
$$

As before, our foliation is made up of surfaces of constant $t$, with boundary $S_{t}$ given by $r=R$. To apply the energy expression (96), we need the following ingredients,

$$
N=\sqrt{V}, \quad N^{i}=0, \quad \widehat{K}^{i}=\frac{\sqrt{V}}{R} E^{i}, \quad \widehat{H}^{i}=0, \quad \widehat{\Omega}^{j k}=\frac{1}{R^{2}} E^{j} \wedge E^{k} .
$$

Now use the useful formula (77) in (96), to derive the energy,

$$
E=-\kappa \Omega_{3} R^{3} \sqrt{V}\left\{\frac{6}{R} \Delta(\sqrt{V})+\frac{24 \alpha}{R^{3}}\left[\Delta(\sqrt{V})-\frac{1}{3} \Delta(V \sqrt{V})\right]\right\}
$$

To keep things tidy, we write

$$
V=y^{2}-m, \quad \bar{V}=y^{2}-\bar{m}
$$


where

$$
y^{2}=1+\frac{R^{2}}{4 \alpha}, \quad m=\sqrt{\frac{\mu}{2 \alpha}}, \quad \bar{m}=\sqrt{\frac{\bar{\mu}}{2 \alpha}} .
$$

Now for large $y$,

$$
\begin{aligned}
\sqrt{V} & =y\left[1-\frac{m}{2 y^{2}}+\mathcal{O}\left(\frac{1}{y^{4}}\right)\right] \\
\Delta(\sqrt{V}) & =-y\left[\frac{\Delta m}{2 y^{2}}+\frac{\Delta\left(m^{2}\right)}{8 y^{4}}+\mathcal{O}\left(\frac{1}{y^{6}}\right)\right] \\
\Delta(V \sqrt{V}) & =-3 y^{3}\left[\frac{\Delta m}{2 y^{2}}-\frac{\Delta\left(m^{2}\right)}{8 y^{4}}+\mathcal{O}\left(\frac{1}{y^{6}}\right)\right] .
\end{aligned}
$$

If we plug this back into (129) we find,

$$
E=3 \kappa \Omega_{3}\left[2 \alpha \Delta\left(m^{2}\right)\right]+\mathcal{O}\left(\frac{1}{y^{2}}\right)
$$

Now we send $R$, or equivalently $y$, to infinity, to derive the black hole mass,

$$
M=3 \kappa \Omega_{3} \Delta \mu
$$

If we had chosen the background to be the black hole of zero size, we would have $\bar{\mu}=2 \alpha$. Our black hole mass would be given by $M=3 \kappa \Omega_{3}(\mu-2 \alpha)$, which agrees with the "minisuperspace" method employed in [57.

\section{Discussion}

In this paper, we have derived a neat and easy to use expression for the gravitational energy of a solution in Gauss-Bonnet gravity. This was done using a Hamiltonian approach, much like the one used by Hawking and Horowitz [5] for Einstein gravity. Given the technical complexity of the derivation, our final expression (96) is remarkably simple.

There have been other ways of calculating the energy of certain Gauss-Bonnet solutions [57, 14, 15]. We have shown that our Hamitonian approach yields results that are consistent with these. Each approach has its advantages and disadvantages, as we will now discuss.

Consider the "conserved charge" method given in [14, 15]. The authors identify a conserved current associated with a timelike Killing vector. The gravitational energy corresponds to the "charge" of this current. This method can be applied to generic higher derivative gravities, of which Gauss-Bonnet gravity is just a special case. However, the background spacetimes, or vacua, are always assumed to be maximally symmetric everywhere. That is not to say that this method cannot be extended to a more general choice of background. This should clearly be a topic for future re-

search. It would also be interesting to know how to apply this method to the special case discussed in section 3.2 . 
Similarly, we should also ask if we can extend our Hamiltonian approach to more general higher derivative gravities. It should be fairly easy to consider the Lovelock action [16],

$$
S=\sum_{n=0}^{\left[\frac{D-1}{2}\right]} \alpha_{n} S_{n}, \quad S_{n}=\int_{\mathcal{M}} \Omega^{A_{1} B_{1}} \wedge \ldots \wedge \Omega^{A_{n} B_{n}} \wedge e_{A_{1} B_{1} \ldots A_{n} B_{n}} .
$$

Here we have a linear combination of the first $\left[\frac{D-1}{2}\right]$ Euler characters, with the surface terms given by the same combination of Chern-Simons forms [18]. Life would be more difficult if we wanted to consider an arbitrary combination of Riemann tensors, as the surface terms are generally unknown ${ }^{5}$.

The "minisuperspace" method used in [57] is closest in spirit to the Hamiltonian approach. The idea is to consider a static, spherically symmetric ansatz for the metric, and insert it back into the action. The action becomes one-dimensional, making it easier to fix the boundary term. When we turn to the Hamiltonian, and evaluate it on one of the black hole solutions given in sections 3.1.1 and 3.2, we derive the black hole mass. This method is very simple and easy to use, but somewhat limited. It can only be applied when the one-dimensional "minisuperspace" model is valid. This is OK for the black hole spacetimes discussed in [57], but a more general approach is clearly desirable.

The Hamiltonian approach developed in this paper is the appropriate generalisation. It can be applied whatever the symmetries of the solution, and without having to reduce the number of dimensions. In particular, we will use it to investigate the generalisation of braneworld holography [55] for Gauss-Bonnet gravity [56].

To sum up, we have derived an expression (96) for the energy of a solution to Gauss-Bonnet gravity. This can be applied whatever our choice of background, and whatever the symmetries of our solution. This should, hopefully, give us a platform to investigate Gauss-Bonnet gravity more thoroughly.

\section{Acknowledgements}

I would like to thank James Gregory for many interesting discussions throughout this entire project. I would also like to thank him, and Simon Ross, for proof reading this article. Thanks also go to Christos Charmousis for introducing me to GaussBonnet gravity during a very long viva! Final thanks go to Steven Gerrard and Michael Owen for scoring the goals that beat United. AP was funded by PPARC.

\section{A Rewriting $S_{\text {kinetic }}$}

We begin with the expression for $S_{\text {kinetic }}$ given in equation (41). This contains terms like $\$_{\perp} H^{a}$, which need to be eliminated by integration by parts. Using the fact that

$$
\$_{\perp} \zeta_{a_{1} \ldots a_{m}}=\$_{\perp} E^{a_{m+1}} \wedge \zeta_{a_{1} \ldots a_{m+1}},
$$

\footnotetext{
${ }^{5}$ Attempts have been made to derive surface terms for general higher derivative gravities using auxiliary fields 72 , 73, 74. However, in the GB case, these results do not agree with [18, so one should proceed with caution. A deeper understanding of boundary terms versus boundary conditions can be found in 75$]$.
} 
we find

$$
\begin{aligned}
S_{\text {kinetic }}= & \kappa \int_{\mathcal{M}} E^{\perp} \wedge\left\{\pi_{a} \wedge \$_{\perp} E^{a}+4 \alpha\left[H^{b} \wedge \$_{\perp} \tilde{\Omega}^{c d}-\tilde{\nabla} H^{b} \wedge \$_{\perp} \tilde{\omega}^{c d}\right] \wedge \zeta_{b c d}\right\} \\
& -\kappa \int_{\Sigma_{\infty}} 2 H^{b} \wedge \zeta_{b}+4 \alpha H^{b} \wedge \Lambda^{c d} \wedge \zeta_{b c d}
\end{aligned}
$$

where $\pi_{a}$ is given by equation (48) and $\Lambda^{c d}$ by equation (49).

If we note that $\theta^{\perp b}=-H^{b}$ is the only non-zero component of $\theta^{A B}$ on $\Sigma_{\infty}$, we can use equations (8) and (18) to show that,

$$
S_{\infty}=\kappa \int_{\Sigma_{\infty}} 2 H^{b} \wedge \zeta_{b}+4 \alpha H^{b} \wedge \Lambda^{c d} \wedge \zeta_{b c d}
$$

This will cancel off the second line in equation (139).

We will soon need the following identities,

$$
\tilde{\nabla} \zeta_{a_{1} \cdots a_{m}} \equiv 0 \equiv \tilde{\nabla} \tilde{\Omega}^{c d}
$$

The left hand side follows automatically from the zero-torsion condition (21), whereas the right hand side is just the frame-form version of the Bianchi identity. We now make use of (141), and the relation,

$$
E^{\perp} \wedge \$_{\perp} \tilde{\Omega}^{c d}=d t \wedge \tilde{\nabla}\left(N \$_{\perp} \tilde{\omega}^{c d}\right)
$$

to show that,

$$
\begin{aligned}
\int_{\mathcal{M}} E^{\perp} \wedge\left[H^{b} \wedge \$_{\perp} \tilde{\Omega}^{c d}-\tilde{\nabla} H^{b} \wedge \$_{\perp} \tilde{\omega}^{c d}\right] \wedge \zeta_{b c d} \\
=\int d t \int_{\Sigma_{t}}\left[H^{b} \wedge \tilde{\nabla}\left(N \$_{\perp} \tilde{\omega}^{c d}\right)-\tilde{\nabla} H^{b} \wedge N \$_{\perp} \tilde{\omega}^{c d}\right] \wedge \zeta_{b c d} \\
=-\int d t \int_{\Sigma_{t}} \tilde{d}\left[N H^{b} \wedge \$_{\perp} \tilde{\omega}^{c d} \wedge \zeta_{b c d}\right] \\
=\int d t \int_{S_{t}} N H^{b} \wedge \$_{\perp} \tilde{\omega}^{c d} \wedge \zeta_{b c d}
\end{aligned}
$$

Note that we have applied Stokes' Theorem in the following way. If $\tilde{A}$ is a $(D-2)$-form on $\Sigma_{t}$, then

$$
\begin{aligned}
\int d t \int_{\Sigma_{t}} \tilde{d} \tilde{A}=\int_{\mathcal{M}} d t \wedge \tilde{d} \tilde{A} & =\int_{\mathcal{M}} d t \wedge d \tilde{A} \\
& =-\int_{\mathcal{M}} d(d t \wedge \tilde{A})=-\int_{B} d t \wedge \tilde{A}=-\int d t \int_{S_{t}} \tilde{A}
\end{aligned}
$$

where we have used the fact that $d \tilde{A}=\tilde{d} \tilde{A}+E^{\perp}\{\cdots\}$ [64. We now insert (140) and (143) into equation (139) to give,

$$
S_{\text {kinetic }}=\kappa \int d t \int_{\Sigma_{t}} \pi_{a} \wedge N \$_{\perp} E^{a}+\kappa \int d t \int_{S_{t}} 4 \alpha N H^{b} \wedge \$_{\perp} \tilde{\omega}^{c d} \wedge \zeta_{b c d}-S_{\infty} .
$$


Notice that $\$_{\perp} \tilde{\omega}^{c d}$ has been removed from the bulk part of $S_{\text {kinetic }}$. This is because we have zero torsion. $\tilde{\omega}^{c d}$ is not an independant dynamical variable, so any time derivatives of it should indeed disappear from the bulk.

We are not yet finished with $S_{\text {kinetic }}$. Equation (145) still contains derivatives of the shift vector. From equation (14), we deduce that

$$
\$_{\perp} E^{a}=\frac{1}{N}\left(\dot{E}^{a}-\$_{\vec{N}} E^{a}\right), \quad \vec{N}=N^{a} \frac{\partial}{\partial x^{a}}=E^{a}{ }_{b} N^{b} X_{a} .
$$

Since $\vec{N}$ lives entirely on $\Sigma_{t}, \$_{\vec{N}}$ is just the intrinsic Lie derivative on $\Sigma_{t}$ 64. Therefore,

$$
\$_{\vec{N}} E^{a}=i_{\vec{N}}\left(\tilde{d} E^{a}\right)+\tilde{d}\left(i_{\vec{N}} E^{a}\right)=-N^{b} i_{\left[\frac{\partial}{\partial x^{b}}\right]}\left(\tilde{w}_{c}^{a}\right) E^{c}+\tilde{\nabla}\left(E_{b}^{a} N^{b}\right) .
$$

where $i_{Y} A$ is the interior product of the vector $Y$ and the $p$-form $A$ [76]. After some integration by parts we see that,

$$
\int d t \int_{\Sigma_{t}} \pi_{a} \wedge \$_{\vec{N}} E^{a}=\int d t \int_{\Sigma_{t}} N^{a} \mathcal{H}_{a}-\int d t \int_{S_{t}}(-1)^{D} N^{a} \pi_{b} E_{a}^{b}
$$

where the momentum constraint, $\mathcal{H}_{a}$, is given by equation (150). In deriving this constraint, we have used the fact that,

$$
\pi^{b} \wedge E^{c}=\pi^{c} \wedge E^{b}
$$

This is not obvious but can be shown using the symmetries of $H_{a b}$ and the Riemann tensor $^{6}$ (on $\left.\Sigma_{t}\right)$.

By inserting equation (148) into (145), we arrive at the final expression (47) for $S_{\text {kinetic }}$

\section{References}

[1] R. Arnowitt, S. Deser, and C. Misner in Gravitation: An Introduction to Current Research, L. Witten, ed. New York, Wiley, 1962.

[2] L. F. Abbott and S. Deser, "Stability of gravity with a cosmological constant," Nucl. Phys. B195 (1982) 76.

[3] J. D. Brown and J. York, James W., "Quasilocal energy in general relativity," gr-qc/9209012.

[4] J. D. Brown and J. York, James W., "Quasilocal energy and conserved charges derived from the gravitational action," Phys. Rev. D47 (1993) 1407-1419.

[5] S. W. Hawking and G. T. Horowitz, "The Gravitational Hamiltonian, action, entropy and surface terms," Class. Quant. Grav. 13 (1996) 1487-1498, gr-qc/9501014.

\footnotetext{
${ }^{6}$ The Riemann tensor on $\Sigma_{t}$ is given by $\tilde{R}_{b c d}^{a}$ where $\tilde{\Omega}^{a}{ }_{b}=\frac{1}{2} \tilde{R}_{b c d}^{a} E^{c} \wedge E^{d}$.
} 
[6] J. D. Brown, S. R. Lau, and J. York, James W., "Energy of Isolated Systems at Retarded Times as the Null Limit of Quasilocal Energy," Phys. Rev. D55 (1997) 1977-1984, gr-qc/9609057.

[7] J. D. Brown, S. R. Lau, and J. W. York, "Canonical Quasilocal Energy and Small Spheres," Phys. Rev. D59 (1999) 064028, gr-qc/9810003.

[8] J. D. Brown, S. R. Lau, and J. W. York, "Action and Energy of the Gravitational Field," gr-qc/0010024.

[9] S. A. Hayward, "Quasilocal gravitational energy," Phys. Rev. D49 (1994) $831-839, \mathrm{gr}-\mathrm{qc} / 9303030$

[10] S. A. Hayward, "Gravitational energy in spherical symmetry," Phys. Rev. D53 (1996) 1938-1949, gr-qc/9408002.

[11] N. Pinto-Neto and I. D. Soares, "The Gravitational energy in asymptotically anti-de Sitter space-times," Phys. Rev. D52 (1995) 5665-5669.

[12] S. Bose and N. Dadhich, "On the Brown-York quasilocal energy, gravitational charge, and black hole horizons," Phys. Rev. D60 (1999) 064010, gr-qc/9906063.

[13] S. A. Hayward, "Gravitational energy as Noether charge," gr-qc/0004042.

[14] S. Deser and B. Tekin, "Gravitational energy in quadratic curvature gravities," Phys. Rev. Lett. 89 (2002) 101101, hep-th/0205318.

[15] S. Deser and B. Tekin, "Energy in generic higher curvature gravity theories," hep-th/0212292.

[16] D. Lovelock, "The Einstein tensor and its generalizations," J. Math. Phys. 12 (1971) 498-501.

[17] C. Lanczos Ann. Math. 39 (1938) 842.

[18] R. C. Myers, "Higher derivative gravity, surface terms and string theory," Phys. Rev. D36 (1987) 392.

[19] F. W. Hehl, J. Nitsch, and P. Von der Heyde, "Gravitation and Poincare gauge field theory with quadratic Lagrangian,". Einstein Commemorative Volume, Plenum Press, 1979/80.

[20] A. Jakubiec and J. Kijowski, "On theories of gravitation with nonlinear Lagrangians," Phys. Rev. D37 (1988) 1406-1409.

[21] J. P. Duruisseau and R. Kerner, "The effective gravitational Lagrangian and the energy momentum tensor in the inflationary universe," Class. Quant. Grav. 3 (1986) 817-824. 
[22] F. Mueller-Hoissen, "Spontaneous compactification with quadratic and cubic curvature terms," Phys. Lett. 163B (1985) 106-110.

[23] F. Mueller-Hoissen, "Gravity actions, boundary terms and second order field equations," Nuc. Phys. B337 (1990) 709-736.

[24] K. S. Stelle, "Renormalization of higher derivative quantum gravity," Phys. Rev. D16 (1977) 953-969.

[25] P. Candelas, G. T. Horowitz, A. Strominger, and E. Witten, "Vacuum configurations for superstrings," Nucl. Phys. B258 (1985) 46-74.

[26] M. B. Green and P. Vanhove, "D-instantons, strings and M-theory," Phys. Lett. B408 (1997) 122-134, hep-th/9704145.

[27] B. Zwiebach, "Curvature squared terms and string theories," Phys. Lett. B156 (1985) 315.

[28] B. Zumino, "Gravity theories in more than four-dimensions," Phys. Rept. 137 (1986) 109.

[29] J. Madore, "Kaluza-Klein theory with the Lanczos Lagrangian," Phys. Lett. A110 (1985) 289.

[30] J. Madore, "On the nature of the initial singularity in a Lanczos cosmological model," Phys. Lett. A111 (1985) 283-284.

[31] N. Deruelle and J. Madore, "Kaluza-Klein cosmology with the Lovelock Lagrangian,". In *Liege 1986, Proceedings, Origin and early history of the universe* $^{*}$ 277-284. (see Conference Index).

[32] J. Madore, "Cosmological applications of the Lanczos Lagrangian," Classical and Quantum Gravity 3 (1986), no. 3, 361-371.

[33] B. Giorgini and R. Kerner, "Cosmology in ten-dimensions with the generalized gravitational Lagrangian," Class. Quant. Grav. 5 (1988) 339-351.

[34] C. Charmousis and J.-F. Dufaux, "General Gauss-Bonnet brane cosmology," Class. Quant. Grav. 19 (2002) 4671-4682, hep-th/0202107.

[35] P. Binetruy, C. Charmousis, S. C. Davis, and J.-F. Dufaux, "Avoidance of naked singularities in dilatonic brane world scenarios with a Gauss-Bonnet term," Phys. Lett. B544 (2002) 183-191, hep-th/0206089.

[36] S. C. Davis, "Generalised Israel junction conditions for a Gauss-Bonnet brane world," hep-th/0208205.

[37] E. Gravanis and S. Willison, "Israel conditions for the Gauss-Bonnet theory and the Friedmann equation on the brane universe," hep-th/0209076. 
[38] C. Germani and C. F. Sopuerta, "String inspired braneworld cosmology," Phys. Rev. Lett. 88 (2002) 231101, hep-th/0202060.

[39] C. Germani and C. F. Sopuerta, "Varying fundamental constants from a string-inspired brane world model," hep-th/0210086.

[40] S. Nojiri, S. D. Odintsov, and S. Ogushi, "Friedmann-Robertson-Walker brane cosmological equations from the five-dimensional bulk (A)dS black hole," Int. J. Mod. Phys. A17 (2002) 4809-4870, hep-th/0205187.

[41] J. E. Lidsey, S. Nojiri, and S. D. Odintsov, "Braneworld cosmology in (anti)-de Sitter Einstein-Gauss- Bonnet-Maxwell gravity," JHEP 06 (2002) 026, hep-th/0202198.

[42] S. Nojiri, S. D. Odintsov, and S. Ogushi, "Cosmological and black hole brane world universes in higher derivative gravity," Phys. Rev. D65 (2002) 023521, hep-th/0108172.

[43] S. Nojiri and S. D. Odintsov, "Brane-world cosmology in higher derivative gravity or warped compactification in the next-to-leading order of AdS/CFT correspondence," JHEP 07 (2000) 049, hep-th/0006232.

[44] K. A. Meissner and M. Olechowski, "Brane localization of gravity in higher derivative theory," Phys. Rev. D65 (2002) 064017, hep-th/0106203.

[45] I. P. Neupane, "Consistency of higher derivative gravity in the brane background," JHEP 09 (2000) 040, hep-th/0008190.

[46] I. P. Neupane, "Gravitational potential correction with Gauss-Bonnet interaction," Phys. Lett. B512 (2001) 137-145, hep-th/0104226.

[47] I. P. Neupane, "Localized gravity with higher curvature terms," Class. Quant. Grav. 19 (2002) 5507-5523, hep-th/0106100.

[48] Y. M. Cho and I. P. Neupane, "Warped brane-world compactification with Gauss-Bonnet term," hep-th/0112227.

[49] Y. M. Cho and I. P. Neupane, "Anti-de sitter black holes, thermal phase transition and holography in higher curvature gravity," Phys. Rev. D66 (2002) 024044, hep-th/0202140.

[50] Y. M. Cho, I. P. Neupane, and P. S. Wesson, "No ghost state of Gauss-Bonnet interaction in warped background," Nucl. Phys. B621 (2002) 388-412, hep-th/0104227.

[51] L. Randall and R. Sundrum, "A large mass hierarchy from a small extra dimension," Phys. Rev. Lett. 83 (1999) 3370-3373, hep-ph/9905221.

[52] L. Randall and R. Sundrum, "An alternative to compactification," Phys. Rev. Lett. 83 (1999) 4690-4693, hep-th/9906064. 
[53] P. Horava and E. Witten, "Heterotic and type I string dynamics from eleven dimensions," Nucl. Phys. B460 (1996) 506-524, hep-th/9510209.

[54] J. P. Gregory and A. Padilla, "Exact braneworld cosmology induced from bulk black holes," Class. Quant. Grav. 19 (2002) 4071-4083, hep-th/0204218.

[55] A. Padilla, "Braneworld cosmology and holography," hep-th/0210217.

[56] J. P. Gregory and A. Padilla, "Braneworld holography in Gauss-Bonnet gravity," hep-th/0304250

[57] J. Crisostomo, R. Troncoso, and J. Zanelli, "Black hole scan," Phys. Rev. D62 (2000) 084013, hep-th/0003271.

[58] G. W. Gibbons and S. W. Hawking, "Action integrals and partition functions in quantum gravity," Phys. Rev. D15 (1977) 2752-2756.

[59] T. Eguchi, P. B. Gilkey, and A. J. Hanson, "Gravitation, gauge theories and differential geometry," Phys. Rept. 66 (1980) 213.

[60] Y. Choquet-Bruhat, C. DeWitt-Morette, and M. Dillard-Bleick, Analysis, Manifolds and Physics. North-Holland, New York, 1982.

[61] S. R. Lau, "Differential forms and wave equations for general relativity," Int. J. Mod. Phys. D7 (1998) 857, gr-qc/9612020.

[62] R. M. Wald, General Relativity, ch. 10. The University of Chicago Press, 1984.

[63] C. Teitelboim and J. Zanelli, "Dimensionally continued topological gravitation theory in Hamiltonian form," Class. Quant. Grav. 4 (1987) L125-L129.

[64] J. Isenberg and J. Nester, "Canonical gravity," in General Relativity and Gravitation, A. Held, ed., vol. 1, pp. 23-97. 1981.

[65] L. P. Eisenhart, Riemannian Geometry, ch. IV. Princeton University Press, 1964.

[66] J. Louko, J. Z. Simon, and S. N. Winters-Hilt, "Hamiltonian thermodynamics of a Lovelock black hole," Phys. Rev. D55 (1997) 3525-3535, gr-qc/9610071.

[67] D. G. Boulware and S. Deser, "String generated gravity models," Phys. Rev. Lett. 55 (1985) 2656.

[68] M. Banados, C. Teitelboim, and J. Zanelli, "Dimensionally continued black holes," Phys. Rev. D49 (1994) 975-986, gr-qc/9307033.

[69] R. C. Myers, "Black holes in higher curvature gravity," gr-qc/9811042.

[70] R.-G. Cai, "Gauss-Bonnet black holes in AdS spaces," Phys. Rev. D65 (2002) 084014, hep-th/0109133. 
[71] R.-G. Cai and K.-S. Soh, "Topological black holes in the dimensionally continued gravity," Phys. Rev. D59 (1999) 044013, gr-qc/9808067.

[72] S. Nojiri and S. D. Odintsov, "Finite gravitational action for higher derivative and stringy gravities," Phys. Rev. D62 (2000) 064018, hep-th/9911152.

[73] S. Nojiri and S. D. Odintsov, "Anti-de Sitter black hole thermodynamics in higher derivative gravity and new confining-deconfining phases in dual CFT," Phys. Lett. B521 (2001) 87-95, hep-th/0109122.

[74] M. Cvetic, S. Nojiri, and S. D. Odintsov, "Black hole thermodynamics and negative entropy in deSitter and anti-deSitter Einstein-Gauss-Bonnet gravity," Nucl. Phys. B628 (2002) 295-330, hep-th/0112045.

[75] J. M. Pons, "Boundary conditions from boundary terms, Noether charges and the trace K Lagrangian in general relativity," Gen. Rel. Grav. 35 (2003) $147-174$, gr-qc/0105032

[76] M. Nakahara, Geometry, Topology and Physics, ch. 5. Institute of Physics Publishing, 1990. 